\title{
Correction to: Studying Weariness Prediction Using SMOTE and Random Forests
}

\author{
Yu Weng, Fengming Deng, Guosheng Yang, Liandong Chen, \\ Jie Yuan, Xinkai Gui, and Jue Wang
}

Correction to:

Chapter "Studying Weariness Prediction Using SMOTE and Random Forests" in: M. Qiu (Ed.):

Smart Computing and Communication, LNCS 11344, https://doi.org/10.1007/978-3-030-05755-8_39

In the original version of this chapter, a wrong project number was stated in the Acknowledgements Section. This has now been corrected. 